\section{EDUCATION}

Research, Innovation and Solutions on-line ${ }^{(2)}$
PSYCHOLOGY

I+D+i
Electronic Journal of Research

in Educational Psychology

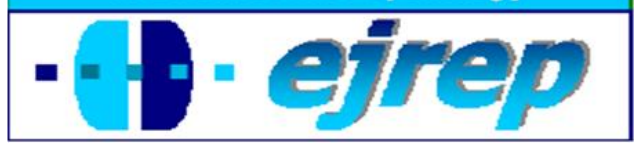

Editorial EOS

\title{
Conocer las emociones a través de juegos: Ayuda para los futuros docentes en la toma de decisiones
}

\section{Pere Lavega ${ }^{1}$, Gemma Filella ${ }^{2}$, Maria Jesús Agulló ${ }^{2}$, Anna Soldevila ${ }^{2}$, Jaume March $^{3}$}

\footnotetext{
${ }^{1}$ INEFC (Instituto Nacional de Educación Física), Universidad de Lleida, ${ }^{2}$ Departamento de Pedagogía y Psicología, Universidad de Lleida, ${ }^{3}$ Jaume March Institut de Recerca Biomédica de Lleida IRBLleida, Universidad de Lleida,
}

\section{España}




\section{Resumen}

Introducción. El objetivo de este estudio fue proporcionar orientaciones para ayudar a profesionales de la educación física a tomar decisiones en torno a las emociones que produjeron diferentes juegos deportivos clasificados en cuatro dominios de acción motriz (psicomotor, cooperación, oposición y cooperación/oposición).

Método. La muestra correspondió a 284 estudiantes universitarios de educación física y de educación primaria (INEFC, Facultad de Educación, Universidades de Lleida y de Barcelona, España). A través de un diseño cuasi-experimental los estudiantes indicaron en un cuestionario validado la intensidad sentida en trece emociones tras participar en cada juego. El análisis de los datos se realizó a través de los árboles de clasificación.

Resultados. La comparación de los resultados en los diferentes dominios de acción motor mostró que los juegos cooperativos fueron los que activaron emociones positivas más intensas entre los estudiantes. En los juegos no competitivos de cooperación los resultados en emociones positivas fueron $18.3 \%$ más elevados que en los juegos competitivos.

Conclusión. Los resultados sugieren que el tipo de juegos a elegir es la primera decisión importante que debería plantearse para educar emociones en los estudiantes. Cada dominio de acción motriz está asociado a la producción de diferentes tipos de emoción.

Palabras Clave: Educación física, Juegos, Emociones, Dominios de acción motriz, Praxiología motriz. 


\title{
Understanding emotions through games: Helping trainee teachers to make decisions
}

\begin{abstract}
Introduction. The aim of this study was to provide guidelines to help professionals make decisions regarding the types of emotions produced by different sporting games classified into four domains of motor action (psychomotor, co-operation, opposition and cooperation/opposition).

Method. The sample comprised 284 first-year university students of physical education and primary education (INEFC, Faculty of Education, universities of Lleida and Barcelona, Spain) aged between. Through a quasi-experimental design the students indicated, on a valided questionnaire, the intensity felt for thirteen emotions after play games. A classification tree was generated in order to analyse the data.
\end{abstract}

Results. Comparison of different motor action domains showed that cooperative games were the most likely to elicit intense positive emotions among students. In non-competitive cooperation games, scores for positive emotions were $18.3 \%$ higher than those reported for competitive games.

Conclusions and Conclusions: The findings suggested that the type of games chosen is the first important decision to make in terms of educating students' emotions. Each domain of motor action is associated with the production of certain types of emotion.

Keywords: Physical education, Games, Emotions, Motor action domains, Motor Praxeology. 


\section{Introducción}

Una de las principales contribuciones de la educación física reside, precisamente, en la capacidad de presentar a los estudiantes actividades que les permitan experimentar diferentes clases de emociones. Sin embargo, muchos educadores siguen creyendo que el verdadero valor de la educación física (EF) se encuentra sólo en sus aspectos más racionales, pasando por alto la importancia de experimentar emociones positivas, negativas y ambiguas. De hecho, la experiencia humana se debe reconocer como emocional y social, en tanto que activa al mismo tiempo la parte cognitiva y física, lo que significa que pensamos, sentimos, actuamos e interactuamos simultáneamente ante cualquier tipo de experiencia (Denzin, 1984; Dewey, 1938; Fernández, 2008; Parlebas, 2001, Pena \& Repetto, 2008).

Han trascurrido más de cuarenta años desde que Parlebas indicó, por primera vez, que en la afectividad se encuentra la clave de la conducta motriz, argumentando que la estructura cognitiva y la acción motriz están modeladas por la afectividad. Según Parlebas (2001), la EF desempeña una función primordial en la educación de la conducta motriz de cada estudiante. El concepto de conducta motriz implica considerar a la persona que toma decisiones motrices, reconocer sus reacciones, su noción de riesgo y sus estrategias corporales, así como la manera de interpretar cada respuesta motriz de los demás participantes. Cualquier conducta motriz no sólo revela la participación estrictamente física del jugador, sino también da testimonio de la experiencia personal que le acompaña (por ejemplo, la felicidad, los temores, las percepciones, las emociones, etc.). Desde este punto de vista, en la conducta motriz se refleja realmente la manera de ser y de sentir de cada persona.

Los apartados siguientes proporcionan argumentos teóricos que justifican la importancia de introducir la educación de las emociones en la formación de conocimiento pedagógico del contenido en los profesores de EF. Resulta fundamental que el profesorado de EF pueda adquirir conocimientos científicos dirigidos a predecir qué tipo de emociones pueden activarse por cada familia de juegos deportivos. Por ello, en los dos últimos apartados de la introducción se revisan los fundamentos teóricos de la praxiología motriz, desarrollando los conceptos de lógica interna de los juegos deportivos y de dominios de acción motriz. Se muestra cómo los juegos deportivos se pueden clasificar en diferentes dominios de acción motriz empleando criterios científicos. Se trata de una herramienta de enseñanza extraordinaria dado que cada 
dominio de acción motriz activa diferentes tipos de experiencias motrices y provoca distintos niveles de intensidad para cada clase de emociones positivas, negativas o ambiguas.

\section{El conocimiento pedagógico del contenido (CPC)}

En 1987 se definió el CPC como un concepto integrado por contenido y pedagogía, entendido como la orientación básica que caracteriza las intervenciones de los educadores, es decir, como la manera que lleva al educador a concebir de un modo concreto la profesión. El CPC identifica los rasgos diferenciadores de aquellos conocimientos que son necesarios para la enseñanza (Shulman y Richert, 1988). Para McCaughtry (2004) y McCaughtry y Rovegno (2003) las concepciones más actuales de los profesores del CPC llevan a aplicar la noción de Dewey al currículum de los estudiantes. Esta concepción destaca la idea de que los profesores deben ser capaces de considerar el trasfondo en el que acontecen los aprendizajes de los estudiantes si desean ser competentes en el ejercicio de enseñar (e.g. Grossman, 1990; Schempp, Manross, Tan, \& Fincher, 1998; Bunker \& Thorpe, 1997; Werner, Thorpe, \& Bunker, 1996).

La revisión de la literatura muestra que muchos investigadores del CPC se han centrado casi exclusivamente en identificar cómo los educadores evalúan las cualidades físicas y cognitivas de las experiencias de los estudiantes, ignorando la comprensión de las emociones que sienten sus alumnos e integrándola en el proceso de enseñanza. Sin embargo, en los últimos años, algunos estudiosos han afirmado que la emoción es fundamental en la corriente del CPC dado que los educadores para tomar decisiones útiles en su labor pedagógica deben comprender cómo se producen las vivencias emocionales de los estudiantes en el proceso de aprendizaje. Estas vivencias deben relacionarse con el tema específico que se esté tratando, así como evaluarse en relación con los otros estudiantes, y con el contexto cultural en el que acontecen (véase por ejemplo, Hargreaves, 1998; Hargreaves, 2000; Hollingsworth, Dybdahl, y Minarik, 1993). De hecho, se desprende que las decisiones que toman algunos profesores respecto el contenido, el currículum y las estrategias pedagógicas a utilizar se hayan mediatizados por la manera en la que éste interpreta las emociones (Hargreaves, 1998; McCaughtry, 2004; Rosiek, 2003; Zemby-las, 2002).

La Lógica Interna. Aquello que da a los juegos su identidad. Todos los juegos deportivos se inscriben en un dominio particular de acción motriz que exige a los jugadores una forma específica de participación y una manera específica de relacionarse con el espacio, con objetos, con el tiempo y con otros jugadores. Estas distintas formas de acción motriz que ca- 
racterizan a una determinada práctica motriz, se corresponden con lo que Parlebas (2001) denomina "la lógica interna" de la situación.

Cada juego desencadena conductas motrices originales, unidas a combinaciones de nuevas acciones motrices y a estrategias diferentes. Parece que cada vivencia lúdica desencadena acciones muy dispares que aparentemente conducen a los protagonistas a un desorden impredecible. Sin embargo, bajo este supuesto desorden se encuentra una organización profunda, que ordena a las acciones de juego de acuerdo a las regularidades que establece la lógica interna de esa actividad. Cada juego o deporte puede ser concebido como un sistema praxiológico (Lagardera y Lavega, 2003; Lagardera y Lavega, 2004), con una lógica interna que impone un sistema de obligaciones y lleva a sus participantes a establecer un tipo particular de relaciones establecidas por las reglas de ese juego deportivo.

Dominios de acción motriz y emociones. Parlebas (2001) se sirve del concepto de lógica interna y emplea la teoría de sistemas para elaborar una clasificación sistémica de los juegos y deportes. La aplicación del criterio de interacción motriz, le lleva a distinguir cuatro familias de juegos o dominios de acción motriz. Cada dominio de acción motriz hace emerger diferentes tipos de relaciones y, por tanto, distintas consecuencias en sus jugadores. Los juegos que pertenecen a un mismo dominio de acción motriz comparten rasgos comunes en su lógica interna, ya que tienden a activar características específicas de la personalidad de los participantes.

(a) Juegos psicomotores en los cuales los participantes intervienen sin poder interactuar con ningún compañero o rival. Estos juegos requieren eficacia, la medición de la fuerza física, el auto-descubrimiento y conocimiento del cuerpo, y ayudan a los participantes a identificar sus virtudes y defectos. El salto de longitud y lanzamiento de un objeto a una diana son ejemplos de esta categoría.

(b) Juegos cooperativos, en los que diferentes jugadores se ayudan mutuamente para alcanzar un objetivo común. Estos juegos activan el diálogo social y el acuerdo o pacto respetuoso con los otros participantes. Bailar con un compañero o pasar la pelota entre varias personas evitando que caiga al suelo son ejemplos de esta categoría. 
(c) Juegos de oposición, en los cuales los jugadores deben oponerse a uno o más rivales que muestran intereses opuestos. En estos juegos los participantes deben tomar decisiones, anticipar sus acciones, descifrar las acciones de los contrarios y actuar estratégicamente. Juegos de persecución y deportes como el judo y el tenis simple pertenecen a esta categoría.

(d) Juegos de cooperación/oposición, en los cuales varios jugadores que forman parte de un equipo desafían a los contrarios generalmente organizados en equipos. Son ejemplos de esta categoría los deportes como el fútbol, el baloncesto, el balonmano y otros juegos de equipo.

Cada uno de estos cuatro dominios de acción motriz puede realizarse con o sin competición, es decir, con o sin victoria final. Cuando existe competición, los jugadores se clasifican en ganadores y perdedores, cosa que no ocurre en los juegos que no tienen un final establecido.

\section{Clasificación de las emociones}

Este estudio emplea la clasificación de las emociones de Bizquera, que a su vez se basa en la categorización que hace Lazarus al distinguir las emociones en positivas, negativas y neutras (Lazarus, 1991; Lazarus, 2000). No obstante, con el ánimo de adaptar la terminología a nuestro contexto se ha sustituido el término 'neutro' por 'ambiguo'. De este modo se distinguen tres tipos de emociones: positivas (alegría, humor, amor y felicidad), negativas (ira, ansiedad, miedo, tristeza, rechazo y vergüenza) y ambiguas (sorpresa, esperanza y compasión) (Bizquera, 2000).

Cualquier profesor de educación física dispone de los medios necesarios para decidir qué tipo de juego o deporte quiere emplear para desarrollar las diferentes emociones en sus estudiantes. Por este motivo, el presente estudio pretende ofrecer una herramienta que ayude a los profesores de educación física a elegir los juegos deportivos más adecuados para alcanzar sus objetivos pedagógicos de acuerdo con las emociones que estas prácticas motrices activan. 


\section{Objetivos e hipótesis}

\section{Objetivos}

- Proporcionar directrices para ayudar a los profesionales a tomar decisiones sobre los diferentes tipos de emociones que producen los distintos juegos deportivos.

- Identificar la relación existente entre los juegos deportivos basados en los diferentes dominios de acción motriz y la expresión de emociones positivas y negativas.

- Identificar la relación entre los juegos deportivos competitivos y no competitivos y la expresión de emociones positivas y negativas.

\section{Hipótesis}

- Juegos deportivos basados en los diferentes dominios de acción motriz podrían crear circunstancias que activen valores intensos en la expresión de emociones positivas y valores de intensidad moderada en la vivencia de emociones negativas.

- Los profesores que pretendan activar emociones positivas como la felicidad, la alegría, el humor, la empatía, la solidaridad o la calma, podrían tomar la decisión más adecuada a partir de la selección de juegos de determinados dominios de acción motriz.

- Los profesores podrían reducir la intensidad en la vivencia de emociones negativas como el odio, la ansiedad, la frustración, la inseguridad o rechazo, mediante el uso de juegos no competitivos.

\section{Método}

\section{Participantes}

La muestra estuvo formada por 284 estudiantes universitarios, 212 alumnos de la licenciatura en educación física (INEFC, universidades de Lleida y Barcelona, España) y 72 estudiantes de la diplomatura de educación primaria (Universidad de Lleida, España). La media de edad fue 19.7 años (SD = 2.63); $144(50.7 \%)$ estudiantes eran chicos y $140(49.3 \%)$ chicas.

Todos los estudiantes dieron su consentimiento para participar en esta investigación. El estudio se realizó en primer lugar en la Universidad de Lleida, concretamente en el centro del Instituto Nacional de Educación física de Cataluña (INEFC) y en la Facultad de Ciencias de la Educación, en las asignaturas de primer curso de teoría y práctica del juego motor y educación social respectivamente. Posteriormente la investigación se realizó en el INEFC, en 
su centro de la Universidad de Barcelona, también en la asignatura de primer curso de teoría y práctica del juego motor.

\section{Medidas}

Una revisión exhaustiva de la literatura especializada en juegos deportivos y emociones constató la ausencia de instrumentos que permitieran relacionar las emociones positivas, negativas y ambiguas, identificadas por autores como Lazarus, (1991, 2000) o Bizquera (2000) con los cuatro dominios de acción motriz y la presencia o ausencia de competición (Parlebas, 2001). Por este motivo se destinaron dos años de trabajo para desarrollar un instrumento que incluyera diferentes variables independientes (dominios de acción motriz: psicomotor, cooperación, oposición, cooperación/oposición) y variables dependientes correspondientes a la intensidad de los diferentes tipos de emociones (emociones positivas: alegría, humor, amor y felicidad; miedo, ansiedad, ira, tristeza, rechazo y vergüenza; y emociones ambiguas: sorpresa, esperanza y compasión). El cuestionario también incluía variables referidas al género y a la experiencia deportiva de los participantes.

El cuestionario se elaboró a través de un trabajo cooperativo entre especialistas del Grupo Internacional de Juegos Deportivos y del Grupo de Investigación en Orientación Psicopedagógica especializado en la enseñanza de competencias emocionales. Este instrumento constaba de las siguientes partes: (a) datos de identificación de los participantes; (b) clasificación de cada situación de juego, (c) diferentes emociones: positivas, negativas y ambiguas. Teniendo en cuenta que cada emoción puede estar asociada a otros términos que funcionan como sinónimos la parte inferior de la hoja incluía una lista de términos relacionados con cada emoción. Al alumnado se le pidió que tras realizar la actividad calificará cada emoción en una escala de 0 a 10 dependiendo de la intensidad con que se había experimentado cada juego, (d) dominio de acción motriz al que correspondía la experiencia deportiva: identificación del deporte practicado en uno de los cuatro dominios de acción motriz, (e) nivel de competición de la experiencia deportiva: deporte de competición local, competición nacional o internacional (ver Anexo 1). Las propiedades psicométricas del cuestionario fueron:

Aceptabilidad. En primer lugar, se evaluó la calidad de los datos considerando la integridad de los registros y la distribución de los valores. Para cada pregunta, se calcularon los efectos techo y suelo, la asimetría y la curtosis. Debido a las características de los ítems, el 
comportamiento fue distinto entre las clases de emociones. Las emociones negativas mostraron un efecto suelo superior al $30 \%$, las emociones ambiguas originaron un efecto suelo en torno al $20 \%$, mientras que no apareció efecto suelo para las emociones positivas. No se observó efecto techo. En las emociones negativas y ambiguas, no se observaron resultados significativos en asimetría y curtosis asociados al efecto suelo.

Fiabilidad. La consistencia interna se calculó a través del alfa de Cronbach; los valores fueron favorables para toda la escala $(\mathrm{n}=271 ; \alpha=.92)$. Se obtuvieron resultados similares para cada tipo de emociones: positivas $(\alpha=.93)$, negativas $(\alpha=.90)$ y ambiguas $(\alpha=.95)$.

La validez de constructo. Los resultados obtenidos mediante el análisis factorial confirmatorio utilizado para probar la estructura del cuestionario mostró que sólo las emociones ambiguas (esperanza, sorpresa, compasión) correspondieron a un único factor. Emociones positivas y negativas estaban vinculadas a varios factores. Se observó que las emociones positivas dependían principalmente de los dominios de acción motriz, y amor correspondía a un sólo factor. Las emociones negativas parecen necesitar más factores, debido a que se consideraron seis emociones negativas. La ansiedad, la tristeza y la vergüenza estaban asociadas a factores por separado. En resumen, el análisis factorial reproduce la estructura del cuestionario de forma adecuada. El modelo final, que constaba de doce factores, mostró una índice de ajuste satisfactorio $(\chi 2=7125.79, \mathrm{df}=4.174)$.

\section{Procedimiento}

El procedimiento seguido en este estudio incluyó las siguientes fases:

\section{Formación en emociones a los estudiantes}

Los participantes recibieron cuatro horas y treinta minutes de formación teórica y práctica en emociones. Se realizaron dos sesiones de una hora y treinta minutos para explicar los principios teóricos más representativos de la educación emocional: concepto, componentes, clasificación y significado de las emociones en el contexto de la educación física. En estas sesiones, el alumnado aprendió a identificar las trece emociones mediante ejercicios correspondientes a situaciones de juego. Se les presentó la descripción ficticia de diferentes situaciones de juego y tuvieron que imaginar qué emociones podrían sentir en cada situación, respondiendo el cuestionario de manera similar a cómo lo realizaron posteriormente en las sesio- 
nes con juegos deportivos. Durante las dos semanas siguientes, se realizaron cuatro sesiones prácticas de una hora y media para identificar las trece emociones (positivas, negativas y ambiguas) en las diferentes situaciones de juego, así como para familiarizarse con las características del cuestionario que iban a usar. El trabajo de campo de este estudio se realizó una vez se constató que el alumnado no tenía dudas para identificar las emociones.

\section{Selección y aplicación de juegos deportivos}

Se seleccionaron dos juegos representativos de cada uno de los dominios de acción motriz, uno competitivo que distinguía a los jugadores en ganadores y perdedores y otro no competitivo.

1) Psicomotor Competitivo. Tira, contacta y gana. Los jugadores se colocan por parejas a unos $2 \mathrm{~m}$ de distancia, dejando un objeto en el suelo en medio de ambos participantes. Cada jugador lanza la bola con la intención de golpear el objeto. Se obtiene un punto cada vez que se consiga. Al final gana quien sume mayor cantidad de puntos.

2) Psicomotor No-competitivo. Conoce tu vientre. Cada jugador recorre con una bola el vientre, explorando las diferentes zonas de esta parte del cuerpo, de acuerdo con las instrucciones del profesor.

3) Cooperación Competitivo. Pasa y gana. Cada equipo se coloca en círculo. Los jugadores deben pasarse entre si diversas pelotas al mismo tiempo. Gana el equipo que es capaz de realizar mayor número de pases.

4) Cooperación No competitivo. Cadenas posteriores (dar un masaje en la espalda del compañero). Por parejas, mientras un jugador flexiona rodillas y desciende el cuerpo el otro le masajea suavemente la espalda presionando y recorriendo con la pelota ambos lados de la columna vertebral. Posteriormente se intercambian los papeles.

5) Oposición Competitivo. Pelota ganadora (simples). Dos jugadores se sitúan a ambos lados de una red e intentan que la pelota golpee dos veces en el campo del rival. Cada vez que el adversario no puede devolver la pelota se obtiene un punto. Al final gana quien sume mayor número de tantos. 
6) Oposición No competitivo. Perseguir imitando. Uno de los participantes se desplaza por la pista de la manera que quiera llevando una pelota de tenis. El resto de jugadores, también con una pelota de tenis, deben imitar los desplazamientos del perseguidor. Cuando se captura a un jugador se intercambian los papeles y se vuelve a empezar.

7) Cooperación-Oposición Competitivo. Pelota ganadora (equipos). Las reglas son las mismas que la pelota ganadora (simples), pero en este caso cada equipo está formado por dos jugadores.

8) Cooperación/Oposición No competitivo. Pelota sentada. Juego paradójico en el que los jugadores pueden pasar la pelota a cualquier jugador mediante un rebote en el suelo (acción de cooperación) o directamente por el aire (acción de oposición). El jugador que recibe el balón con rebote sigue vivo, pero si recibe un pase aéreo entonces queda capturado y debe sentarse. Los capturados se salvan si interceptan la pelota y la pasan a otro jugador que también esté capturado. Todos los jugadores deciden libremente cooperar u oponerse, sin seguir ningún criterio lógico.

Los participantes fueron distribuidos en grupos de treinta alumnos. Los juegos fueron dirigidos por el mismo profesor para todos los grupos, siguiendo las mismas instrucciones. Todas las sesiones de juegos se realizaron en las mismas condiciones. Cada grupo realizó cinco juegos en cada dos sesiones de 90 minutos.

Los juegos introducidos en la primera sesión fueron: 1. Pasa y gana; 2. Pelota ganadora (simples); 3. Pelota ganadora (equipos); y 4. Cadenas posteriores. En la segunda sesión se realizaron los siguientes juegos: 5. Perseguir imitando; 6. Pelota sentada; 7. Tira, contacta y gana; y 8. Conoce tu vientre.

\section{Registro de los datos}

Cada estudiante dispuso de un cuestionario y un bolígrafo. Al inicio el profesor explicaba el juego que los estudiantes debían realizar. Después de participar en cada juego los estudiantes se dirigían a rellenar el cuestionario, indicando la intensidad con la que habían experimentado las diferentes emociones, en una escala de 0 a 10 , en la que 0 significaba que no se había sentido esa emoción y 10 que se había sentido con la máxima intensidad. El cuestionario se respondía individualmente (ver Apéndice 1). 


\section{Análisis de los Datos}

Se generó un árbol de clasificación CHAID (detector de interacción automática de Chi-cuadrado) con el fin de permitir particiones de más de dos ramas, en las que una de las principales variables tenía tres categorías. Se empleó el módulo de Answer-Tree® SPSS Árboles de Clasificación TM 13.0. Se aplicó un sistema validación cruzada del sistema y se prolongó hasta considerar 50 sujetos como número mínimo de casos en los nodos terminales.

Otras características fueron: control de tamaño del árbol (tamaño mínimo de nodo tamaño de divisiones: 10; niveles máximos de árbol: 3), método de validación (10 veces validación cruzada), divisiones iniciales (no establecido), probabilidades a priori para la variable "categorías" de destino (uso de la distribución de frecuencias en el conjunto de datos), costes de clasificación errónea (unitaria para todas las categorías), y manipulación de datos perdidos (uso de predictores sustitutos).

La variable dependiente, es decir, la intensidad emocional (rango 0 -10), fue recodificada en cuatro categorías con el fin de obtener frecuencias similares: 0 - 0.99 (29\%), 1 a 2.99 (24.5\%), 3 a $4.99(17.4 \%)$ y 10.05 (28.6 \%). En el procedimiento CHAID se eligieron los factores: dominios de acción motriz, emoción, competición, dominios de acción motriz de la experiencia deportiva, nivel de competición de la experiencia deportiva.

\section{Resultados}

En relación a la predicción de la tendencia que tienen los juegos deportivos para elicitar diferentes tipos de emociones, el análisis de datos mediante árboles de clasificación mostró los siguientes resultados (ver figuras 1,2 y 3 ).

\section{Primera decisión: tipos de emociones (nodos 1, 2 y 3)}

La primera distinción a tener en cuenta $(\mathrm{p}=.000$ y $\chi 2=2908.7, \mathrm{df}=2)$ corresponde a los tres tipos de emociones (positivas, negativas y ambiguas) asociadas a los nodos (1, 2 y 3 ). Los participantes en los juegos deportivos registraron la intensidad más alta de las emociones positivas (5-10) en el 56.1\% de los casos. Por contra, las emociones negativas alcanzaron los 
valores de intensidad más altos en sólo el $9.2 \%$ de los participantes, mientras que las emociones ambiguas mostraron las puntuaciones más altas en el $20.6 \%$ de los casos.

Segunda decisión: emociones positivas y dominios de acción motriz (nodos 4, 5 y 6 )

Los árboles de clasificación muestran que para predecir que tipo de juegos activan emociones positivas se debe considerar el factor correspondiente al tipo de dominio de acción motriz (nodos 4, 5 y 6$),\left(p=.000\right.$ y $\left.\chi^{2}=60,5, \mathrm{df}=1\right)$. Estas emociones alcanzaron sus valores más intensos (de 5 a 10) en el dominio de la cooperación (70.1\%). Los juegos con presencia de adversarios (juegos de oposición y juegos de cooperación/oposición) se comportaron de la misma manera, activando la intensidad más alta en las emociones positivas en el $53.8 \%$ de los participantes. En los juegos psicomotores, los participantes obtuvieron los valores más intensos en menor número de ocasiones (46.7\%).

Si se decide elegir los juegos cooperativos (nodo 5) con el fin de desencadenar emociones positivas, entonces la siguiente decisión a considerar debería ser el factor correspondiente a la competición ( $\mathrm{p}=.000$ y $\chi^{2}=37,3, \mathrm{df}=1$ ). La intensidad de las emociones positivas obtuvo los valores más altos (puntuaciones entre 5 y 10) en los juegos cooperativos sin competición (nodo 14); estos resultados se observaron en el $79.2 \%$ de los casos. Cuando los juegos cooperativos se hicieron con competición (nodo 13), los valores más altos se obtuvieron en menor número de ocasiones (60.9\% de los participantes).

Si se decide elegir juegos deportivos de oposición o cooperación/oposición (nodo 6), entonces también se debería considerar como factor predictor la experiencia deportiva de los participantes deportivos, es decir, qué tipo de deporte ha sido su práctica habitual (nodos 15 y 16) ( $\mathrm{p}=.000$ y $\chi^{2}=37,3$, df = 1). Los jugadores que habían participado regularmente en deportes de equipo, por ejemplo, gimnasia rítmica de conjuntos, natación sincronizada, deportes de oposición como el judo, tenis simple o karate- y deportes de cooperación/oposición como baloncesto, fútbol o balonmano, registraron los valores máximos para las emociones positivas en el $58.2 \%$ de los casos.

Finalmente, si se decide elegir los juegos deportivos psicomotores (nodo 4) para despertar emociones positivas acto seguido se debería volver a considerar la experiencia deportiva como criterio predictivo (nodos 11 y 12) ( $\mathrm{p}=.002$ y $\left.\chi^{2}=14.7, \mathrm{df}=1\right)$. Los participantes 
que habían participado regularmente deportes sociomotores obtuvieron calificaciones más altas con mayor frecuencia $(50.5 \%)$ que los participantes con antecedentes en deportes psicomotores o los participantes que no tenían historial deportivo (39.6\%).

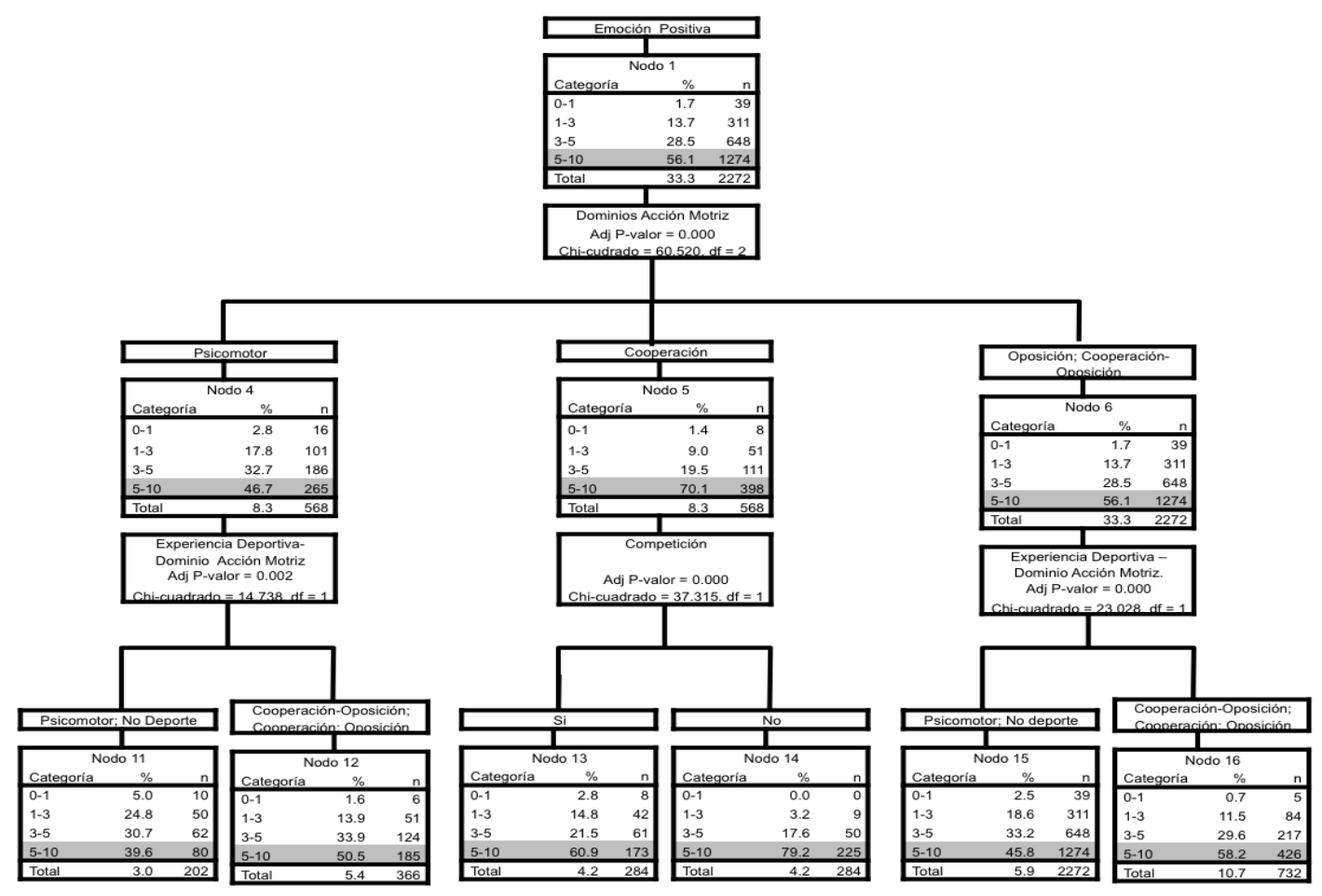

Figura 1. Árbol de clasificación para predecir la enseñanza de emociones positivas en juegos deportivos.

Tercera Decisión: Emociones Negativas y Competición (nodos 7 y 8)

El árbol de clasificación muestra que para predecir que tipos de juegos deportivos elicitan emociones negativas se debería considerar el factor de competición (nodos 7 y 8) (p = .000 y $\left.\chi^{2}=120.3, \mathrm{df}=1\right)$.

Si se eligen juegos deportivos competitivos (nodo 7), entonces las emociones negativas alcanzan sus valores más elevados (de 5 a 10) en el $14.2 \%$ de los participantes. Por contra, si se introducen juegos deportivos no competitivos (nodo 8) estos valores tan solo se observan en el $4.2 \%$ de los casos.

Si se presentan juegos deportivos no competitivos (nodo 8) el siguiente criterio predictor a considerar debería ser el género de los participantes (nodos 19 y 20) $\left(\mathrm{p}=.000\right.$ y $\chi^{2}=18.0$, 
df $=1$ ). En el caso de los chicos los valores más altos surgieron en el $6.2 \%$ de los casos, mientras que en las chicas se registraron únicamente en el $2.1 \%$ de las intervenciones.

Si se eligen juegos deportivos competitivos (nodo 7), el siguiente criterio predictivo a considerar debería ser el nivel de competición en el historial deportivo de los participantes $($ nodos 17 y 18$)\left(\mathrm{p}=0.000\right.$ y $\left.\chi^{2}=26.8, \mathrm{df}=1\right)$. Las emociones negativas alcanzaron los valores más altos en el $15.9 \%$ de los casos cuando los participantes tenían antecedentes de experiencia deportiva en competiciones locales, nacionales o internacionales. Sin embargo, cuando el historial deportivo de los participantes correspondía a una práctica recreativa no competitiva, los valores negativos tan solo aparecieron en el $7.1 \%$ de los casos.

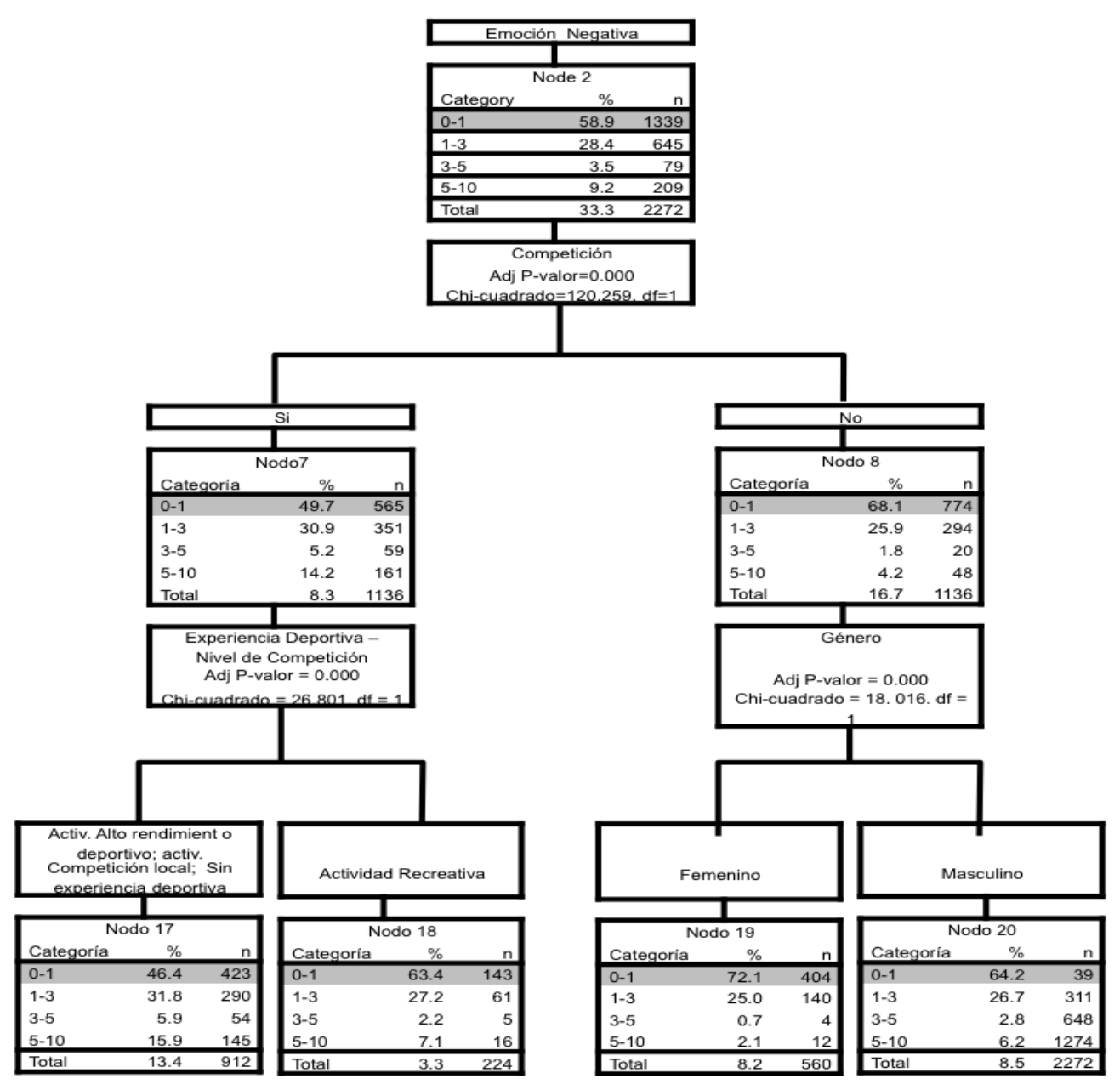

Figura 2. Árbol de clasificación para predecir la enseñanza de las emociones negativas en los juegos deportivos. 
Cuarta Decisión: Emociones Ambiguas y Género (nodos 9 y 10)

El árbol de clasificación muestra que al predecir que tipos de juegos deportivos desencadenan emociones ambiguas se debería considerar el género de los participantes (nodos 9 y 10) $\left(\mathrm{p}=.000\right.$ y $\left.\chi^{2}=76.6, \mathrm{df}=1\right)$.

En juegos deportivos practicados por hombres (nodo 10) $\left(\mathrm{p}=.000 \mathrm{y} \chi^{2}=18.0, \mathrm{df}=1\right)$ la intensidad de las emociones ambiguas alcanzaron los valores más altos (0-5) en el $26.7 \%$ de los casos, mientras que entre las mujeres (nodo 9) estos valores máximos solo se activaron en el $14.2 \%$ de las ocasiones.

Cuando las mujeres participan en los juegos deportivos el siguiente factor predictivo a considerar debería ser el nivel de competición de su experiencia deportiva (nodos 21, 22 y 23) $\left(\mathrm{p}=.000\right.$ y $\left.\chi^{2}=56.7, \mathrm{df}=2\right)$. Las emociones ambiguas alcanzaron los valores máximos en el $19.5 \%$ de las mujeres que habían practicado regularmente deportes en competiciones de nivel local (campeonatos regionales) o de rendimiento (campeonatos nacionales o internacionales). Las mujeres con experiencia en prácticas físicas recreativas (no competitivas y no federadas) expresaron emociones ambiguas de máxima intensidad en el $6.7 \%$ de los casos, mientras que las que nunca habían realizado ejercicio físico obtuvieron esos valores máximos en el $4.2 \%$ de las ocasiones.

Si se pretende desencadenar emociones ambiguas en hombres se debería considerar como factor predictivo la elección de juegos deportivos con o sin competición (nodos 24 y 25) (nodo 10) ( $\left.\mathrm{p}=.000 \mathrm{y} \chi^{2}=22.8, \mathrm{df}=1\right)$. Cuando los juegos no son competitivos los valores más intensos (5-10) se activaron en el 31.9 \% de los casos. En cambio, en los juegos competitivos estas emociones ambiguas registraron los valores máximos en el $21.5 \%$ de los participantes. 


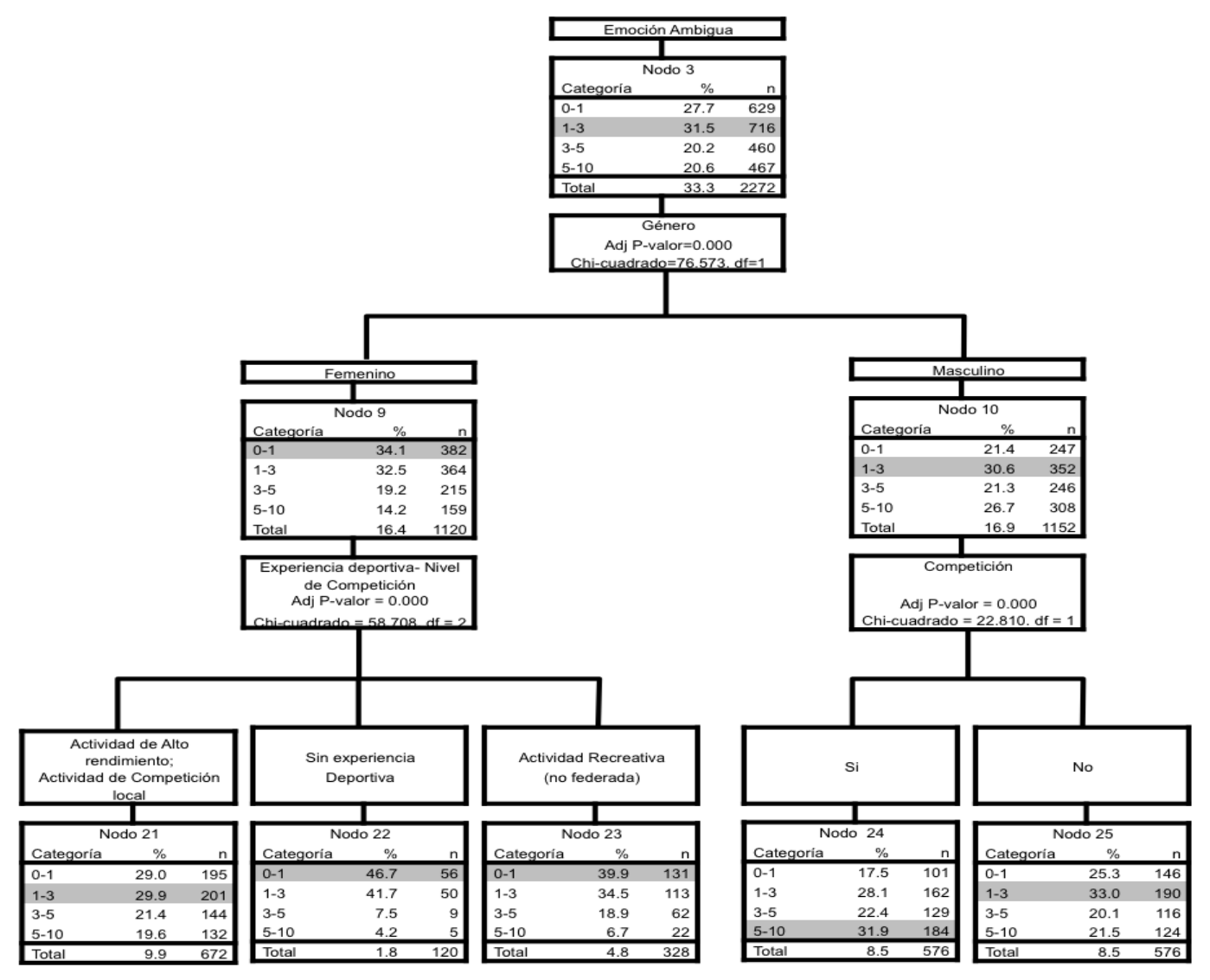

Figura 3. Árbol de clasificación para predecir la enseñanza de las emociones ambiguas en los juegos deportivos.

\section{Discusión}

La discusión se debe centrar en tres principales hallazgos. En primer lugar, los resultados de esta investigación confirman que el uso de los juegos deportivos en educación física es una excelente manera para fomentar experiencias óptimas en los estudiantes. Los resultados también confirman la extraordinaria aportación científica de la Praxiología Motriz o Ciencia de la Acción Motriz para ayudar a los profesores a tomar decisiones con el fin de educar emociones a través de juegos deportivos.

En este sentido se confirma que la actividad física puede promover conductas prosociales y las emociones jugar un papel muy importante, tal y como sido mostrado por Eldar (2008), Parlebas (1999) y Lavega, Mateu, Lagardera, y Filella (2010). Mientras que la literatura muestra que la cognición y el rendimiento han sido factores ampliamente revisados y 
estudiados, el dominio afectivo todavía no ha originado tantas aportaciones. Se han localizado numerosos estudios que prestan la atención sobre aspectos tácticos y técnicos en la enseñanza de los juegos, sin embargo los estudiosos que se dedican a investigar la labor pedagogía y didáctica de los juegos harían bien en considerar las características del alumno que aprende, así como identificar si el alumnado está motivado para seguir practicando actividad física y reconocer la importancia de sus resultados en el ámbito afectivo (Hargreaves, 2000;McCaughtry, 2004; Rosiek, 2003). Todas las corrientes educativas que pretendan aportar procedimientos adecuados en la enseñanza de los juegos, deberían considerar el valor que tiene la vivencia emocional. Esta vivencia repercute directamente en la experiencia de la actividad física que reciben los jóvenes, así como sobre su futura motivación para participar en programas físicos orientados a mejorar su salud psicológica, física y social.

La Praxiología Motriz ofrece sólidas bases teóricas y epistemológicas para el estudio científico de los juegos y deportes (Parlebas, 1999; Lagardera y Lavega, 2003; Lagardera y Lavega, 2004). Dado que los profesores de educación física deben tomar decisiones constantemente con el fin de mejorar la enseñanza de las tareas motrices resulta imprescindible disponer de criterios científicos que les permita elegir las situaciones motrices más apropiadas para cada programa pedagógico (Hargreaves, 1998; Hargreaves, 2000; Hollingsworth et al, 1993; McCaughtry, 2004; Parlebas, 1999) .

El presente estudio confirma que los juegos deportivos basados en los distintos dominios de acción motriz pueden originar aquellas circunstancias asociadas a la activación intensa de emociones positivas. Por contra, los juegos elegidos provocaron muy pocas emociones negativas con valores intensos. Esta conclusión es de gran interés para el campo de la educación física, ya que una de las razones que explica la inactividad de la población adulta es la valoración negativa que se tiene de las experiencias realizadas en la escuela (Daley y O'Gara, 1998). Ruiz, García y Pieron (2008) afirman que los estudiantes que participan en juegos deportivos en edad escolar, así como aquellos que hacen ejercicio físico con regularidad, muestran una actitud mucho más positiva hacia la educación física. Aspectos positivos, tales como la autoestima, la motivación y la actitud emocional correcta promueven el desarrollo y la consolidación de conductas asociadas a una actividad física y a un estilo de vida saludables (Spence y Blanchard, 2001). 
El segundo grupo de hallazgos concierne a la obtención de criterios que pueden ayudar a los profesores de educación física a desencadenar emociones positivas. Esta investigación muestra que la primera decisión importante para activar emociones positivas como la felicidad, la alegría, el humor, la empatía, la solidaridad o la calma corresponde a elegir como criterio predictivo el tipo de juegos o dominio de acción motriz. Esta conclusión confirma resultados obtenidos en otras investigaciones sobre emociones y dominios de acción motriz (Lavega et al., 2010). Se confirma que ningún juego es aséptico ya que todos los juegos deportivos estudiados provocaron reacciones en los participantes. Al considerar los diferentes dominios de acción motriz, los juegos cooperativos y sobre todo los no competitivos, fueron los más apropiados para activar emociones positivas intensas entre los estudiantes. Desde esta óptica, Slutzky y Simpkins (2009) argumentan que las prácticas físicas en grupo mejoran de manera más significativa el concepto de si mismo si se compara con deportes individuales correspondientes al mismo nivel de competición.

Estos resultados concuerdan con afirmaciones de Werner et al. (1996) quienes indican que los primeros propósitos en la enseñanza de cualquier juego no debería ser mejorar únicamente el rendimiento de los estudiantes, sino también favorecer la diversión y participación en los juegos, ya que así se puede promover un estilo de vida más saludable.

El último conjunto de resultados se orientan hacia la necesidad de disponer de criterios para tomar decisiones adecuadas cuando se trata de reducir emociones negativas. Si los profesores no quieren que sus alumnos experimenten altos niveles de emociones negativas como el odio, la ansiedad, la frustración, la culpabilidad, la inseguridad o el rechazo, deben saber que estos valores se pueden reducir si se decide introducir juegos no competitivos, en los que los jugadores no sean divididos en ganadores y perdedores. En este sentido, estamos de acuerdo con Bunker y Thorpe (1997), quienes destacan la importancia de crear un clima positivo para el alumnado, sin que resulte imprescindible juzgar su éxito a partir de si han conseguido realizar sus acciones motrices de manera adecuada o no. La percepción de este buen clima se puede ver mejorada permitiendo que los alumnos decidan si quieren que se tenga en cuenta o no el resultado al practicar juegos competitivos. Bunker y Thorpe (1997) también señalan que mientras las sesiones basadas en las habilidades motrices pueden mostrar al profesor algunos efectos inmediatos en la mejora de estas habilidades, las interacciones motrices y sociales que se originan pueden llevar a los alumnos a que se den cuenta de su falta de habilidad . 
Los profesores actúan de mediadores al relacionar el uso de los juegos deportivos con los resultados educativos o efectos que esperan que sus alumnos alcancen. Por lo tanto, los educadores deberían aprender a tomar decisiones de acuerdo con los resultados que se desean obtener. Si se conoce qué tipo de efectos provocan las tareas motrices de cada dominio de acción motriz, entonces se podrá ayudar al educador a elegir las mejores actividades para promover por ejemplo la solidaridad, la mejora de las relaciones positivas dentro del grupo o si por otra parte se pretende que aprendan a tomar riesgos y a controlar su propia seguridad.

Como conclusión, indicar que numerosos estudios sobre emociones han indicado la necesidad de educarlas con el fin de que las personas puedan llegar a ser independientes y maduras (Bizquera, 2000; Lazarus, 1991; Shoffner, 2009). Los juegos proporcionan situaciones activas que favorecen vivencia de emociones. Por ello permiten abordar el primer paso en la enseñanza de competencias emocionales correspondiente a la toma de conciencia emocional. Una vez la persona es capaz de reconocer las emociones que ha sentido en cada juego deportivo, los profesores pueden podrán pasar a una segunda fase con el objetivo de favorecer la autogestión, la autoestima, el sentido de responsabilidad, una actitud positiva y crítica hacia la vida, una relación más intensa con las otras personas y el bienestar personal.

\section{Agradecimientos}

Los autores mostramos el agradecimiento al apoyo recibido por el Gobierno de España (Dirección General de Investigación, Ministerio de Ciencia e Innovación) al proyecto Perspectiva de género en las emociones suscitadas por los Juegos deportivos psicomotores y de cooperación. [Código DEP2010-21626-C03-01]. También manifestamos el agradecimiento al apoyo obtenido por el Gobierno de Cataluña al proyecto Juegos Deportivos y emociones (AGAURINEFC) [Código 2009SGR1404; VCP/3346/2009].

\section{Referencias}

Bizquera, R. (2000). Educación emocional y bienestar [Emotional education and wellbeing]. Barcelona: Praxis.

Bunker, D., \& Thorpe, R. (1997). A changing focus in games teaching. In L. Almond (Ed.), Physical education in schools (pp. 52-80). London: Kogan Page.

Denzin, N. K. (1984). On understanding emotion. San Francisco: Jossey-Bass. 
Dewey, J. (1938). Experience and education. New York: Collier.

Eldar, E. (2008). Educating through the Physical-behavioural interpretation. Physical Education and Sport Pedagogy, 13(3), 215-229. doi: 10.1080/17408980701345741

Fernandez, P. (2008). La inteligencia emocional en educación [The emotional intelligence in education]. Electronic Journal of Research in Educational Psychology, 15, 6(2), 421436.

Grossman, P. (1990). The making of a teacher. New York: Teachers College Press.

Hargreaves, A. (1998). The emotional practice of teaching. Teaching and Teacher Education, 14(8), 835-854. doi:10.1016/S0742-051X(98)00025-0.

Hargreaves, A. (2000). Mixed emotions: Teachers' perceptions of their interactions with students. Teaching and Teacher Education, 16(8), 811-826.

doi:10.1016/S0742-051X(00)00028-7.

Hollingsworth, S., Dybdahl, M., \& Minarik, L. T. (1993). By chart and chance and passion: The importance of relational knowing in learning to teach. Curriculum Inquiry, 23(1), $5-35$.

Lagardera, F., \& Lavega, P. (2003). Introducción a la praxiología motriz [Introduction to motor praxeology ].Barcelona: Paidotribo

Lagardera, F., \& Lavega, P. (eds). (2004). La ciencia de la acción motriz [The science of motor action]. Lleida: Edicions de la Universitat de Lleida.

Lavega, P., Mateu, M., Lagardera, F., \& Filella, G. (2010) Educar emociones positivas a través de los juegos deportivos [Teaching positive emotions through sporting games]. In M. A. Torralba, M. De Fuentes, J. Calvo, \& J. F. Cardozo (Eds.), Docencia, innovación e investigación en educación física. [Teaching, innovation and research in physical education]. (pp. 111-139). Barcelona: INDE Publicaciones

Lazarus, R. S. (1991). Emotion and adaptation. New York: Oxford University Press.

Lazarus, R. S. (2000). How emotions influence performance in competitive sports. The Sport Psychologist, 14(3), 229-252

McCaughtry, N., \& Rovegno, I. (2003). Development of pedagogical content knowledge: Moving from blaming students to predicting skillfulness, recognizing motor development, and understanding emotion. Journal of Teaching in Physical Education, 22, 355-368. 
McCaughtry, N. (2004). The emotional dimensions of a teacher's pedagogical content knowledge: Influences on content, curriculum, and pedagogy. Journal of Teaching in Physical Education, 23(1), 30-47.

Parlebas, P. (2001). Juegos, deporte y sociedad. Léxico comentado en praxeología motriz [Games, sport and society. An annotated vocabulary of motor praxeology]. Barcelona: Paidotribo.

Pena, M., \& Repetto, E., (2008). Estado de la investigación en España sobre Inteligencia Emocional en el ámbito educativo [State of research in Spain on Emotional Intelligence in education]. Electronic Journal of Research in Educational Psychology, 15, 6(2), 400-420.

Rosiek, J. (2003). Emotional scaffolding: An exploration of the teacher knowledge at the intersection of student emotion and the subject matter. Journal of Teacher Education, 54(5), 399-412. doi: 10.1177/0022487103257089

Ruiz, F., Garcia, M. E., \& Pieron, M. (2008). Physical-sports habits and health. lifestyles of young people and adults. Adapting to Scientific Innovations and Prevailing Social Development. Physical Education Conference in the Town of Ceuta, 13-38.

Schempp, P. G., Manross, D., Tan, S. K. S., \& Fincher, M. D. (1998). Subject expertise and teachers' Knowledge. Journal of Teaching in Physical Education, 17(3), 342-356.

Shoffner, M. (2009). The place of the personal: Exploring the affective domain through reflection in teacher preparation. Teaching and Teacher Education, 25(6), 783-789. doi:10.1016/j.tate.2008.11.012

Shulman, L. S. \& Richert, A. E (1988) The place of the personal: Exploring the affective domain through reflection in teacher preparation. Teaching and Teacher Education, 4(1), 53-62. doi:10.1016/0742-051X(88)90024-8

Slutzky, C. B. \& Simpkins, S. D. (2009). The link between children's sport participation and self-esteem: Exploring the mediating role of sport self-concept. Psychology of Sport and Exercise, 10, 381-389. doi:10.1016/j.psychsport.2008.09.006

Spence, J. C., \& Blanchard, C. (2001). Effect of pretesting on feeling states and self-efficacy in acute exercise. Research Quarterly for Exercise and Sport, 72(3), 310-314.

Werner, P., Thorpe, R., \& Bunker, D. (1996). Teaching games for understanding: Evolution of a model. Journal of Physical Education, Recreation \& Dance, 67(1), 28-33.

Zembylas, M. (2002). Structure of feeling in curriculum and teaching: Theorizing the emotional rules. Educational Theory, 52, 187-208. doi: 10.1111/j.1741-5446.2002.00187.x 
Apéndice 1. Cuestionario Emociones y Dominios de Acción Motriz para Juegos Deportivos

Dominios de Acción Motriz y Emociones

\begin{tabular}{|c|c|c|c|c|c|c|c|c|c|c|c|c|c|c|c|}
\hline Fecha: & Número de estudiante & & & Edad & Género: & Curso: & & & Forn & ción: & $1^{\text {a } S}$ & sión† & Sesión $\uparrow$ & & \\
\hline $\begin{array}{l}\text { Denomi } \\
\text { en difere }\end{array}$ & $\begin{array}{l}\text { del Juego deportivo } \\
\text { ominios de acción motr }\end{array}$ & ibuidas & & Emocio & es Positi & & & & Emocion & Negati & & & Emo & ones $\mathrm{Ar}$ & guas \\
\hline & Nombre del juego & & $\begin{array}{l}\text { Alegria } \\
\text { (Diver- } \\
\text { sión) } \\
\end{array}$ & $\begin{array}{c}\text { Humor } \\
\text { (Reír) }\end{array}$ & $\begin{array}{c}\text { Amor } \\
\text { (Afecto) }\end{array}$ & $\begin{array}{c}\text { Felicidad } \\
\text { (Paz) }\end{array}$ & $\begin{array}{l}\text { Ira } \\
\text { (Enfa- } \\
\text { do) } \\
\end{array}$ & $\begin{array}{l}\text { Miedo } \\
\text { (Terror) }\end{array}$ & $\begin{array}{c}\text { Ansiedad } \\
\text { (preocupa- } \\
\text { ción) } \\
\end{array}$ & $\begin{array}{c}\text { Tristeza } \\
\text { (Frusta- } \\
\text { ción) } \\
\end{array}$ & 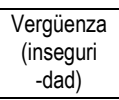 & $\begin{array}{c}\text { Rechazo } \\
\text { (despre- } \\
\text { cio) }\end{array}$ & Esperanza & Sorpresa & Compasión \\
\hline 1. TIRA, & ACTA Y GANA. & Vencedor & & & & & & & & & & & & & \\
\hline & & Perdedor & & & & & & & & & & & & & \\
\hline 2. CONC & J VIENTRE. & & & & & & & & & & & & & & \\
\hline 3. PASA & & Vencedor & & & & & & & & & & & & & \\
\hline & & Perdedor & & & & & & & & & & & & & \\
\hline 4. CADE & OSTERIORES. & & & & & & & & & & & & & & \\
\hline 5. MANC & JIDUAL GANADORA. & Vencedor & & & & & & & & & & & & & \\
\hline & & Perdedor & & & & & & & & & & & & & \\
\hline 6. PERS & R IMITANDO & & & & & & & & & & & & & & \\
\hline 7. MANC & ECTIVA GANADORA. & Vencedor & & & & & & & & & & & & & \\
\hline & & Perdedor & & & & & & & & & & & & & \\
\hline 8. PELO & TADA. & & & & & & & & & & & & & & \\
\hline
\end{tabular}

\section{EMOCIONES POSITIVAS}

Alegría: Entusiasmo, euforia, excitación, contento, diver-

sión, placer, éxtasis, aligeramiento, capricho ...

Humor: Sonrisas, reír, carcajadas,...

Amor: Afecto, cariño, ternura, simpatía, empatía, acepta-

ción, cordialidad, confianza, amabilidad, afinidad,..

Felicidad: Gozo, tranquilidad, paz interior, placidez, satisfacción, bienestar ...

\section{EMOCIONES NEGATIVAS}

Ira: Rabia, cólera, rencor, odio, furia, indignación, enfado, celos, violencia,...

Miedo: Temor, horror, pánico, terror, desasosiego, susto, fobia.

Ansiedad: Angustia, desesperación, inquietud, estrés, preocupación, anhelo, desazón,

Tristeza: Depresión, frustración, decepción, soledad, pena, dolor, desconsuelo,...

Vergüenza: Culpabilidad, timidez, inseguridad, vergüenza ajena, bochorno, pudor.

Rechazo: Hostilidad, desprecio, recelo, menosprecio, asco, repugnancia, antipatía...

\section{EMOCIONES AMBIGUAS}

Sorpresa: Reacción por un imprevisto que puede derivar en emociones +0 -

Esperanza: Consiste en temer lo peor pero anhelar mejorar.

Compasión: Sentirse afectado por el sufrimiento del otro y desear ayudar.p 